\title{
Surface density of the young cluster IC 348 in the Perseus molecular cloud
}

\author{
L. Cambrésy ${ }^{1}$, V. Petropoulou ${ }^{1,2}$, M. Kontizas ${ }^{2}$, and E. Kontizas ${ }^{3}$ \\ 1 Observatoire Astronomique de Strasbourg, 67000 Strasbourg, France \\ e-mail: cambresy@astro.u-strasbg.fr \\ 2 Department of Astrophysics Astronomy \& Mechanics, Faculty of Physics, University of Athens, 15783 Athens, Greece \\ 3 Institute for Astronomy and Astrophysics, National Observatory of Athens, PO Box 20048, 11810 Athens, Greece
}

Received 1 August 2005 / Accepted 1 September 2005

ABSTRACT

The IC 348 young star cluster contains more than 300 confirmed members. It is embedded in the Perseus molecular cloud, making any clustering analysis subject to an extinction bias. In this work, we derive the extinction map of the cloud and revisit the content of IC 348 through a statistical approach that uses the 2MASS data. Our goal was to address the question of the completeness of IC 348 and of young clusters in general. We performed a combined analysis of the star color and density in this region, in order to establish the surface density map of the cluster. We reached the conclusion that IC 348 has structures up to $25^{\prime}$ from the cluster center, and we estimate that about 40 members brighter than $K_{\mathrm{s}}=13 \mathrm{mag}$ are still unidentified. Although we cannot use our statistical method to identify these new members individually, the surface density map gives a strong indication of their actual location. They are distributed in the outer regions of the cluster, where very few dedicated observations have been made so far, which is probably why they escaped previous identification. In addition, we propose the existence of a new embedded cluster associated to the infrared source MSX 6C G160.2784-18.4216, about 38' south of IC 348.

Key words. stars: pre-main sequence - ISM: dust, extinction - open clusters and associations: individual: IC 348

\section{Introduction}

Young stellar clusters concentrate stars in their early stages, making them the privileged location for studying the initial conditions of star formation from the molecular cloud fragmentation to the evolution on the main sequence. Such a large and ambitious enterprise requires the acquisition of multiwavelength data from the millimeter to the X-ray spectral range, in order to probe the different physical phenomena that occur during star formation. The identification of cluster members leads to constrain the cluster history, the initial mass function, and the dynamical evolution. Although the ultimate goal is to take a complete census of the clusters where each object is actually given a membership status, the individual identification is not easy to obtain and is not always necessary. A statistical identification could also lead to information on the cluster morphology and the initial mass function.

The young cluster IC 348 has been investigated regularly for decades and is still a very popular target. Its brightest member is the B5 star BD +31643 , which is part of the Per OB2 association (Blaauw 1952). A distance of $320 \mathrm{pc}$ is usually adopted for the cluster although it is still controversial (Cernis 1993; Herbig 1998; de Zeeuw et al. 1999). Herbig (1954) identified 16 members characterized by their $\mathrm{H} \alpha$ emission, and the current number is about 300 , with a mass and age distribution peak at $0.2 M_{\odot}$ and $2 \mathrm{Myr}$, respectively (Luhman 1999; Carpenter 2002; Luhman et al. 2003; Muench et al. 2003; Preibisch et al. 2003; Preibisch \& Zinnecker 2004; Luhman et al. 2005b). Muench et al. (2003) showed that the extinction reaches values as high as 20 mag toward the cluster line of sight, the cluster itself being only partially embedded in the Perseus molecular cloud with an average extinction of $4.5 \mathrm{mag}$ (Lada \& Lada 1995). The extinction will introduce a bias in the apparent member distribution, as long as the cluster census is incomplete. It is a general characteristic of young clusters to still be associated with their parent molecular cloud. The apparent stellar density, which makes a cluster detectable, results from both the true cluster density and the extinction variation toward its direction. Although a classical clustering analysis is biased in this configuration, it is possible to extract relevant information on clusters with a statistical analysis which takes the extinction into account (Carpenter 2000; Cambrésy et al. 2002).

\section{Cluster mapping}

The Perseus molecular cloud has an elongated and filamentary structure, as shown in the extinction map presented in Fig. 1. 


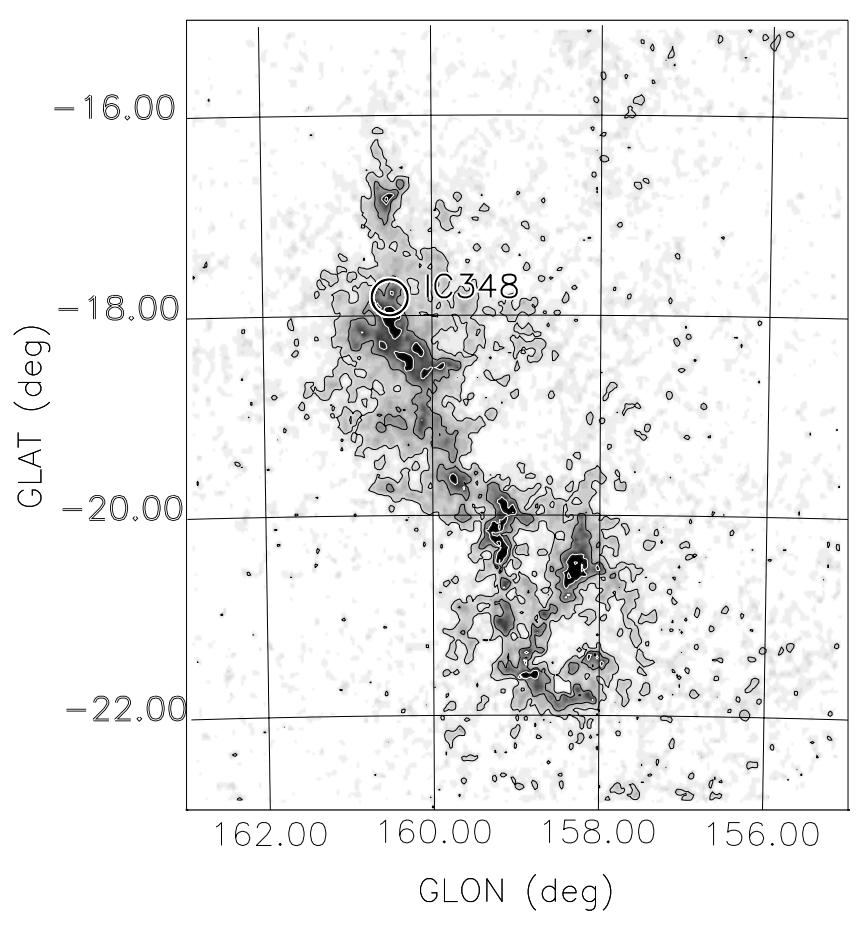

Fig. 1. Perseus extinction map from 2 MASS $H-K_{\mathrm{s}}$ color excess. The spatial resolution is $4^{\prime}$ and isocontours are for $A_{\mathrm{V}}=2,4,8,16$ mag. The circle position and radius are set to contain all the confirmed members of IC 348 .

This map is obtained by measuring the 2MASS $H-K_{\mathrm{s}}$ color excess in cells with adaptive size. The cell size is chosen to contain only 3 stars, and the full map is then convolved by an adaptive kernel to obtain a final uniform spatial resolution of $4^{\prime}$. This technique reduces the non-linear error because of the extinction variations at a smaller scale than the map resolution (Cambrésy et al. 2005). The known members of IC 348 were removed before the extinction map was built.

The final map is very similar to the ${ }^{13} \mathrm{CO}$ map (Padoan et al. 1999) and to the extinction map presented on the COMPLETE webpage $^{1}$ with the NICER method (Lombardi \& Alves 2001). An extinction estimate from reddening or from star counts relies on the assumption that all stars are behind the obscuring cloud and that the stellar population is homogeneous over the whole field. When a young stellar cluster is embedded in a molecular cloud, none of the conditions is fulfilled. The color and the star count maps react differently to the cluster contamination. Cambrésy et al. (2002) proposed using this property to identify clusters by comparing both maps. The next 3 sections describe the technique to build the color map and the count map and to obtain the cluster morphology.

In the following, we focus on a $2^{\circ} \times 2^{\circ}$ field centered on IC 348 at glon $=160.38^{\circ}$, glat $=-17.78^{\circ}$.

\subsection{Reddening}

The technique is based on the pioneering work by Lada et al. (1994), who propose a statistical method to build extinction maps from near-infrared color excesses. The specificity of our

\footnotetext{
${ }^{1}$ http://www.cfa.harvard.edu/COMPLETE/
}

method is to use adaptive cells that contain a fixed number of stars, in order to increase the spatial resolution and to limit the bias due to the inhomogeneous distribution of stars that tend to concentrate in the less obscured part of a cell. A detailed description of this bias can be found in Cambrésy et al. (2005). The visual extinction was obtained through the 2 MASS $H-K_{\mathrm{s}}$ color excess as follows:

$$
\begin{aligned}
E_{H-K_{\mathrm{s}}} & =\left(H-K_{\mathrm{s}}\right)_{\mathrm{obs}}-\left(H-K_{\mathrm{s}}\right)_{\mathrm{int}} \\
A_{\mathrm{V}}^{\mathrm{col}} & =\left(\frac{A_{H}}{A_{\mathrm{V}}}-\frac{A_{K_{\mathrm{s}}}}{A_{\mathrm{V}}}\right)^{-1} E_{H-K_{\mathrm{s}}}
\end{aligned}
$$

where the indices obs and int stand for observed and intrinsic, respectively. Here, $\left(H-K_{\mathrm{s}}\right)_{\text {int }}=0.12 \pm 0.1 \mathrm{mag}$ from the region outside the Perseus cloud. We used the extinction law from Rieke \& Lebofsky (1985), for which $A_{H} / A_{\mathrm{V}}=0.175$ and $A_{K_{\mathrm{s}}} / A_{\mathrm{V}}=0.112$, yielding the color excess to visual extinction conversion formula $A_{\mathrm{V}}=15.87 \times E_{H-K_{\mathrm{s}}}$. The presence of a young embedded cluster will affect the extinction, because 1) YSOs are embedded in the cloud and suffer only a partial obscuration, and 2) YSOs may have different colors than field stars, with an excess at $K_{\mathrm{s}}$ due to an accretion disk for those objects that are younger than about 2 Myr. In the former case, the extinction will be underestimated, in the latter it will be overestimated. The effect does not usually stand out when examining the reddening map because no anomaly (e.g. strong gradient or discontinuity) appears there. Another independent extinction estimation is required to detect the cluster contamination.

\subsection{Star counts}

In the star count method, the decrease in the apparent stellar density is interpreted as a foreground obscuration. It is the historical method for mapping the interstellar extinction (Wolf 1923; Bok 1956). As mentioned above, we used an adaptive technique with the exact same cells as for the color excess mapping. The visual extinction is obtained by assuming the apparent variations of the stellar density are due to foreground obscuration:

$\begin{aligned} A_{K_{\mathrm{s}}}^{\mathrm{cnt}} & =\frac{1}{a} \log \frac{D_{\mathrm{ref}}}{D} \\ A_{\mathrm{V}}^{\mathrm{cnt}} & =\frac{A_{\mathrm{V}}}{A_{K_{\mathrm{s}}}} \times A_{K_{\mathrm{s}}}^{\mathrm{cnt}}\end{aligned}$

where $a$ is the slope of the $K_{\mathrm{s}}$ luminosity function (KLF), $D$ the star density in each cell, and $D_{\text {ref }}$ the density in a reference field. Although the reference star density formally depends on the position (mainly on the galactic latitude), a constant value is correct for small areas of about $1 \mathrm{deg}^{2}$. When a cluster contaminates the field, the stellar density is locally enhanced, and the extinction is biased toward low values. If the local density is higher than the average density over the field ( $\left.D_{\text {ref }}\right)$, which is true if the extinction is small, it gives a negative number for the extinction. Obviously, Eq. (2) is no longer correct, because the measured density actually includes the background stars and the cluster stars: $D=D_{\mathrm{b}}+D_{\mathrm{cl}}$. 


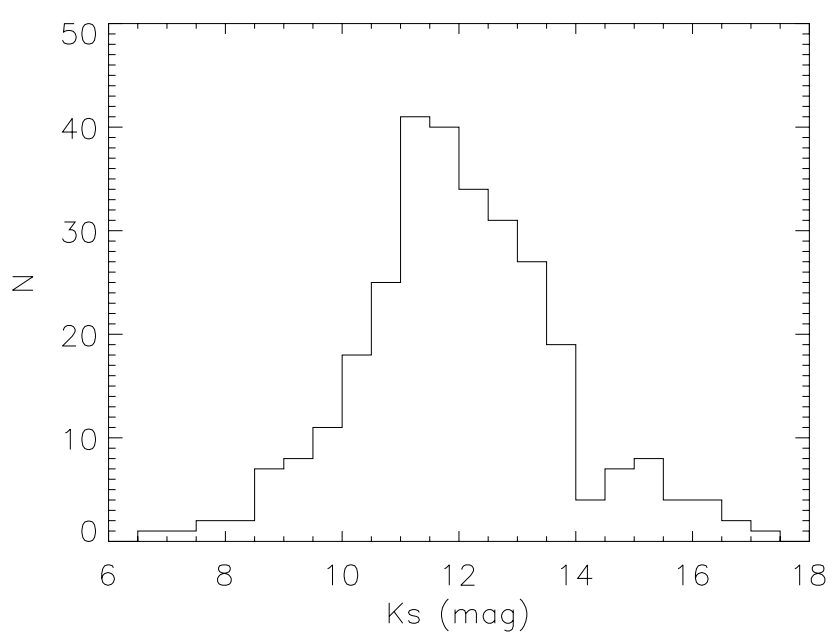

Fig. 2. $K_{\mathrm{s}}$ luminosity function of the 297 stars of the cluster with available $K_{\mathrm{s}}$ magnitude (Luhman et al. 2003, 2005a).

\subsection{Comparison of the two maps}

Comparison of the maps requires the two calibrations to be consistent. The spatial resolution is identical in both maps by construction. The relative calibration of the two maps is obtained using an arbitrary value for $D_{\text {ref }}$ in Eq. (2) and by forcing the median of $\Delta A_{\mathrm{V}}=A_{\mathrm{V}}^{\text {col }}-A_{\mathrm{V}}^{\text {cnt }}$ to zero. The absolute calibration of the extinction maps is not relevant for the cluster study.

The difference $\Delta A_{\mathrm{V}}$ should be a structureless map, if the field is free of any cluster contamination. Otherwise, the cluster morphology will be revealed by the structures contained in the $\Delta A_{\mathrm{V}}$ map because $D_{\mathrm{cl}}>0$, meaning $A_{\mathrm{V}}^{\mathrm{col}}>A_{\mathrm{V}}^{\mathrm{cnt}}$.

\section{Application to the IC $\mathbf{3 4 8}$ young cluster}

\subsection{Data}

The data were extracted from the 2MASS All-Sky Point Source Catalog (Cutri et al. 2003) for the region defined by $158.6^{\circ}<$ glon $<162.2^{\circ}$ and $-19.2^{\circ}<$ glat $<-16.3^{\circ}$. The list of the 304 confirmed members of IC 348 was provided by Luhman et al. (2003) and Luhman et al. (2005a), 278 of which are present in 2MASS. Figure 2 shows the $K_{\mathrm{s}}$ luminosity function of the full member list. The distribution peaks at $K_{\mathrm{s}} \approx 11.5 \mathrm{mag}$ with more than $75 \%$ of the sources brighter than $K_{\mathrm{s}}=13 \mathrm{mag}$. On the other hand, faint stars dominate the background star population with a number of sources proportional to $10^{a \times K_{\mathrm{s}}}$, with $a=0.31$. While a magnitude cut at $K_{\mathrm{s}}<13$ mag keeps $75 \%$ of the cluster members, it removes $75 \%$ of the background stars. In order to increase the signal-to-noise ratio in the $\Delta A_{\mathrm{V}}$ map, we applied this cut before building the reddening and star count maps.

\subsection{Results}

Following the method of Sect. 2, the extinction was from adaptive cells that contain 3 stars. The reddening and the count maps were then smoothed with a Gaussian kernel to reach the final uniform resolution of $8^{\prime}$. We stress that it requires a convolution by an adaptive kernel with $F W H M=\left(8^{2}-F W H M_{\text {orig }}^{2}\right)^{1 / 2}$,

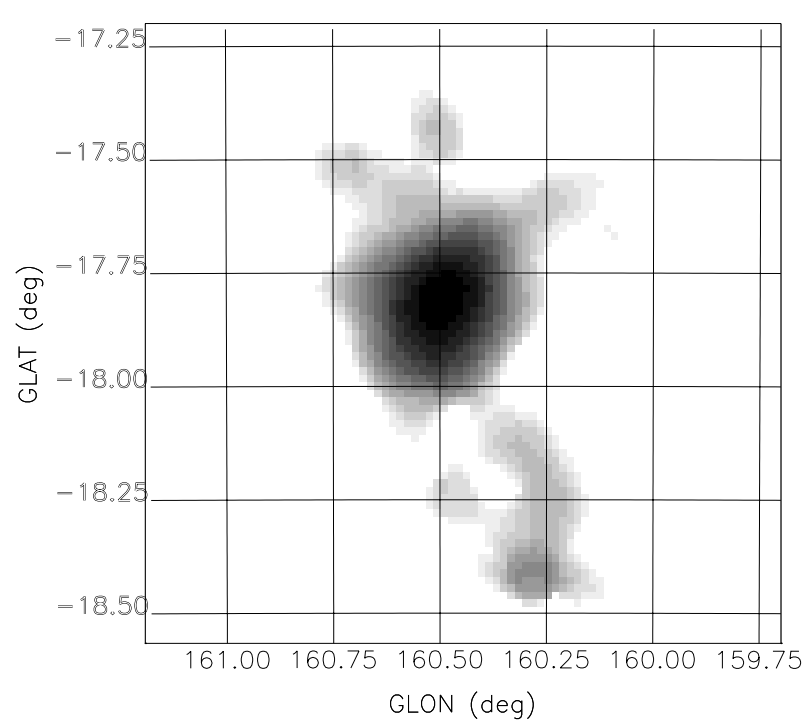

Fig. 3. Surface density map of IC 348 obtained from stars brighter than $K_{\mathrm{s}}=13 \mathrm{mag}$. The map shows the region $2 \sigma=7.7 \mathrm{mag}$ above the noise.

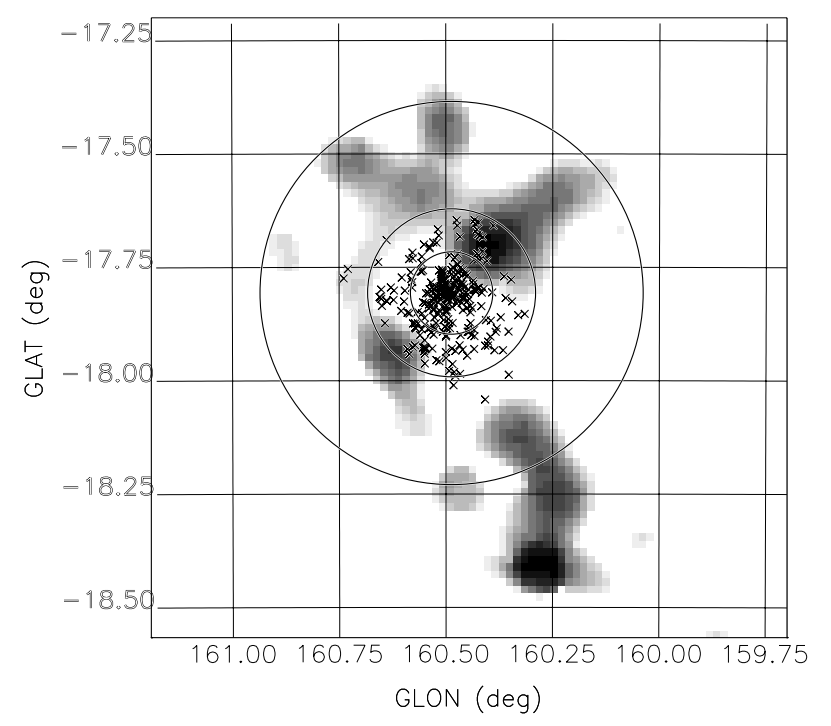

Fig. 4. Same as Fig. 3 but derived from a catalog filtered from all known IC 348 members. These members are overlaid on the map as crosses. Circles at $5^{\prime}, 10^{\prime}$, and $25^{\prime}$ radius define the core (Lada \& Lada 1995), the halo (Muench et al. 2003), and the cluster boundary suggested from our study.

where $F W H M_{\text {orig }}$ is the resolution in arcmin of the original maps. The difference between the maps from Eqs. (1) and (2) is presented in Fig. 3. As expected a peak appears at the IC 348 position.

The very same process was repeated on the 2 MASS catalog filtered from all known IC 348 members. The resulting $\Delta A_{\mathrm{V}}$ map is presented in Fig. 4 with the cluster members overlaid as crosses. Significant structures remain on the map, which strongly supports the idea that the cluster extends beyond the halo defined by Muench et al. (2003) at 10.33' and beyond the ROSAT X-ray detection boundary at $15^{\prime}$ (Preibisch et al. 1996). The map exhibits a faint ring around the central part of the cluster and several substructures. The farthest 
clump, located at $l=160.26 b=-18.42$, is $38^{\prime}$ south of the IC 348 center and seems linked to the main cluster by a ridge. It contains an infrared source reported by IRAS and MSX, MSX6C G160.2784-18.4216 (Kraemer et al. 2003). It is, however, likely that this structure corresponds to another star-formation site in Perseus. Further analysis needs to be carried out to conclude whether it is related to IC 348. Another clump located to the north-west of IC 348 has the particularity of being within the halo radius (Muench et al. 2003) of IC 348, which means this region has already been studied, although several bright members have not been identified spectroscopically in this area.

Figures 3 and 4 were obtained by subtracting two extinction maps, making the pixel unit to be magnitude. It has no physical meaning in this context, except that it goes with the star density. A straightforward way to convert these magnitudes into star density would require the real extinction to be known, but both the reddening and the count maps are contaminated by the YSOs. Fortunately, the outer region of IC 348 has a low star density, since it is the first time the excess has been detected. It means the sample must be dominated by background stars, which makes the $H-K_{\mathrm{s}}$ color excess an acceptable estimation of the extinction. We stress that the median color is used within cells rather than the mean, which ensures a low sensitivity to the YSOs contamination as long as it is less than $50 \%$. Assuming the color-based extinction map is correct, Fig. 4 was converted in surface density as follows:

$D_{\mathrm{cl}}=c \times\left(10^{a \Delta A_{K_{\mathrm{s}}}}-1\right)$

where $c$ is obtained using the knowledge that the difference between $N_{\mathrm{cl}}$ in Figs. 3 and 4 must be exactly the number of confirmed members that were removed from the original 2MASS catalog to make the second figure (i.e. 212 stars for $K_{\mathrm{s}}<13 \mathrm{mag}$ ). We finally conclude that $40 \pm 6$ stars with $K_{\mathrm{s}}<13 \mathrm{mag}$ are responsible for the excess seen in Fig. 4. The quoted uncertainty is the statistical uncertainty associated with the Poisson law followed by the star distribution. A total number of 350 stars brighter than 13 mag at $K_{\mathrm{s}}$ was found in the area, meaning an $11 \%$ level contamination by the cluster, which justifies our choice of relying on the color median as an extinction estimator. Following Eq. (3), the southern clump centered on MSX6C G160.2784-18.4216 would contain about 10 YSOs brighter than $K_{\mathrm{s}}<13 \mathrm{mag}$.

The color-color diagram (Fig. 5) for the stars located in the region defined in Fig. 4 shows almost no star with a $H-K_{\mathrm{s}}$ excess. This is also true for the confirmed IC 348 members, which indicates that most YSOs have already lost their inner accretion disk that is responsible for the infrared excess at $2 \mu \mathrm{m}$. Individual identification is therefore not possible with the 2MASS photometry.

\section{Conclusions}

The statistical comparison of the stellar color and density permit building the surface density map of embedded clusters. We applied our technique to IC 348, using two different sample catalogs, one with all 2MASS sources and the other one filtered

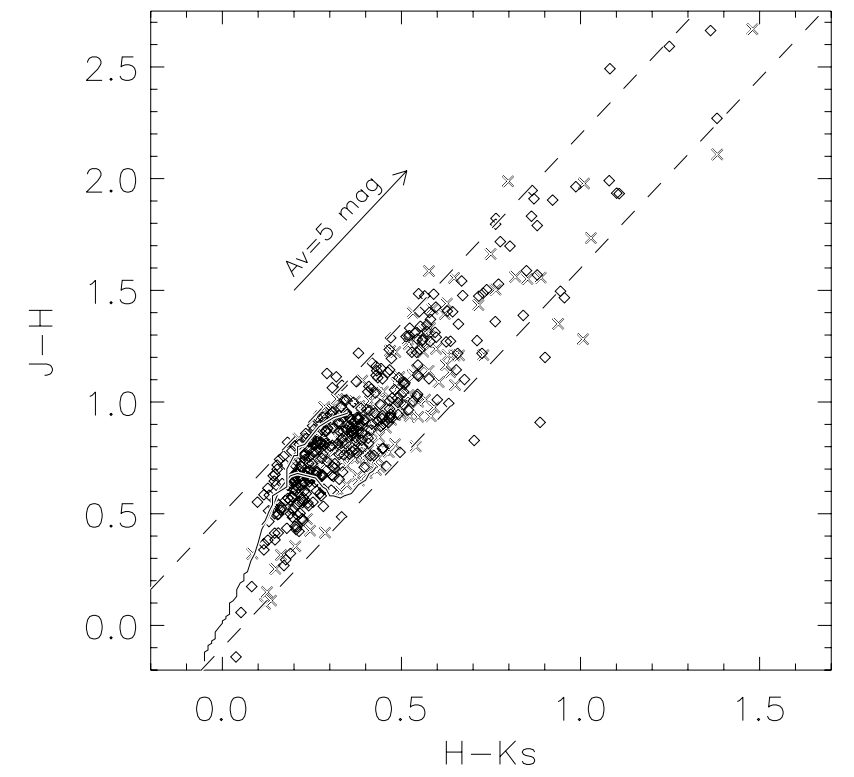

Fig. 5. Color-color diagram for stars with $K_{\mathrm{s}}<13 \mathrm{mag}$ in the area defined by Fig. 4 (diamonds) and for confirmed IC 348 members (crosses). The dashed lines are parallel to the reddening vector.

from all known associated YSOs. As expected, the first map shows a peak at the cluster location, but it is more surprising to find evidence of structures in the filtered map, too. These structures are distributed around IC 348, suggesting they are actually an extension of about 40 new members for the wellknown cluster. This result encourages more detailed study of the structure of IC 348 and, in particular, of its dynamical evolution, as it created these clumps in the cluster. As no infrared excess was found in 2MASS, we cannot identify the potential new members individually. Longer wavelength follow-up with Spitzer may exhibit the presence of a residual disk, but X-ray observations with XMM-Newton are probably more appropriate to finding the older pre-main-sequence stars in the outer region of IC 348. Spectroscopic follow-up remains the most suitable way to obtain the final diagnostic for membership. Proper motion measurements could be an indicator of the membership and would be of great interest for the kinematic study of the cluster.

Acknowledgements. This publication makes use of data products from the Two Micron All Sky Survey, which is a joint project of the University of Massachusetts and the Infrared Processing and Analysis Center/California Institute of Technology, funded by the National Aeronautics and Space Administration and the National Science Foundation.

\section{References}

Blaauw, A. 1952, Bull. Astron. Inst. Netherlands, 11, 405

Bok, B. J. 1956, AJ, 61, 309

Cambrésy, L., Beichman, C. A., Jarrett, T. H., \& Cutri, R. M. 2002, AJ, 123, 2559

Cambrésy, L., Jarrett, T. H., \& Beichman, C. A. 2005, A\&A, 435, 131

Carpenter, J. M. 2000, AJ, 120, 3139 
Carpenter, J. M. 2002, AJ, 124, 1593

Cernis, K. 1993, Baltic Astron., 2, 214

Cutri, R. M., Skrutskie, M. F., van Dyk, S., et al. 2003, Explanatory Supplement to the 2MASS All Sky Data Release

de Zeeuw, P. T., Hoogerwerf, R., de Bruijne, J. H. J., Brown, A. G. A., \& Blaauw, A. 1999, AJ, 117, 354

Herbig, G. H. 1954, PASP, 66, 19

Herbig, G. H. 1998, ApJ, 497, 736

Kraemer, K. E., Shipman, R. F., Price, S. D., et al. 2003, AJ, 126, 1423

Lada, C. J., Lada, E. A., Clemens, D. P., \& Bally, J. 1994, ApJ, 429, 694

Lada, E. A., \& Lada, C. J. 1995, AJ, 109, 1682

Lombardi, M., \& Alves, J. 2001, A\&A, 377, 1023

Luhman, K. L. 1999, ApJ, 525, 466
Luhman, K. L., Stauffer, J. R., Muench, A. A., et al. 2003, ApJ, 593, 1093

Luhman, K. L., Lada, E. A., Muench, A. A., \& Elston, R. J. 2005a, ApJ, 618, 810

Luhman, K. L., McLeod, K. K., \& Goldenson, N. 2005b, ApJ, 623, 1141

Muench, A. A., Lada, E. A., Lada, C. J., et al. 2003, AJ, 125, 2029

Padoan, P., Bally, J., Billawala, Y., Juvela, M., \& Nordlund, Å. 1999, ApJ, 525, 318

Preibisch, T., Zinnecker, H., \& Herbig, G. H. 1996, A\&A, 310, 456

Preibisch, T., Stanke, T., \& Zinnecker, H. 2003, A\&A, 409, 147

Preibisch, T., \& Zinnecker, H. 2004, A\&A, 422, 1001

Rieke, G. H., \& Lebofsky, M. J. 1985, ApJ, 288, 618

Wolf, M. 1923, Astron. Nachr., 219, 185 